\title{
Multi-Phase Signal Control Method For Single Intersection Based On Multi-Person Cooperative Game Theory
}

\author{
Linglong Tan ${ }^{1}$,Yueling Wang ${ }^{2}$, Qing Wang ${ }^{1}$,Yannan Sun ${ }^{1}$, Zexin Li ${ }^{1}$ \\ 1.Electronic communication engineering college, Anhui Xinhua University, Anhui,230088, China \\ 2. AHCNCERT, Anhui Hefei, Hefei,China
}

Keywords:Signal control,Multi people Cooperation Game, Paramics

\begin{abstract}
Study the application of cooperative game algorithm in signal control, by analyzing the game characteristics of four phase signal control of single intersection .In order to maximize the overall interests of the intersection as the control target, build the corresponding game model: Select four strategies which the income is more than zero.It means at the same time, only one phase of the four phase is the green light. Select the intersection in each direction of the vehicle queue length as the game earned value. Establish the income matrix which any phase of the four phase get the right to pass. $\alpha-$ Characteristic function method is used to determine the coalition characteristic function in cooperative games.And then, use the Shapley estimation to allocate the profit of cooperative game.In the end,In the Paramics simulation, select a set of strategies which is closest to the game value as the traffic signal control scheme.
\end{abstract}

\section{Multi person game theory knowledge}

\subsection{Multi-person Cooperative Game Theory}

Game theory ${ }^{[1]}$.It is the decision problem and the equilibrium problem of the rational decision subject.A game theory has three basic elements: bureau of the people, the policy set and the payment function.

A collection of people in the Bureau $N=\{1,2, \ldots \mathbf{n}\}$ : Bureau of the people are involved in the game. There are ' $n$ ' people in the game. It is called the ' $n$ ' game. Strategy set $S_{\mathrm{i}}$ :specific strategies can be limited, but also can be infinite. If the policy set is limited, it is called a fin ite game, otherwise known as infinite game.

Payment function $P_{\mathrm{i}}$ : There is a pay function $P_{\mathrm{i}}(\mathrm{s})$ for every ' $i$ ' in the Bureau. It is a function of the situations, that is, the Bureau of the people ' $i$ ' in the situation under the income of ' $\mathrm{s}$ '.

In the cooperative game called transferable utility $G=[N, v]$, A return $v(S)$ of the League $\mathrm{S}$, said the alliance $S$ to join the major league game opportunity gains, or the alliance ' $S$ ' independent activities to get the maximum benefit. The method of determining the characteristic function of the alliance is ${ }^{[2-5]}$ :

(1)When the alliance $S$ the establishment of the alliance can be obtained independently of the proceeds of the transaction behavior outside of the union $\mathrm{S}, v(S)$ the maximum yield for the union ' $\mathrm{S}$ ' can be obtained.

(2) The ' $S$ ' alliance gains by independent alliance $S$ best effect and benefit people in the block.A strategic non cooperative game $G=\left[N,\left\{X_{i}\right\},\left\{P_{i}\right\}\right]$, The mixed strategy set of participants $i$ is $X_{i}$.Income function is $P_{i}$.The characteristic function can be derived from the strategy game by using the following methods

Method: Rational threat characteristic function In $G=\left[N,\left\{X_{i}\right\},\left\{P_{i}\right\}\right]$, the rational threat characteristic function of alliance ' $S$ ' is defined as:

$$
v(S)=\sum_{i \in S} E_{i}\left(x^{*}, y^{*}\right), v(N \backslash S)=\sum_{j \in N \backslash S} E_{j}\left(x^{*}, y^{*}\right)
$$

Among them $x^{*} \in X_{S}, y^{*} \in X_{N \backslash S}$, it is determined by the Nash equilibrium point $\left(x^{*}, y^{*}\right)$ of the two person zero sum game

$G^{\prime}=\left[(S, N \backslash S),\left\{X_{S}, X_{N \mid S}\right\},\left\{\sum_{i \in S}^{\prime} E_{i}(x, y)_{i}-\sum_{j \in N \mid S}^{\prime} E_{j}(x, y), \sum_{j \in N \mid S}^{\prime} E_{j}(x, y)-\sum_{i \in S}^{\prime} E_{i}(x, y)\right\}\right]$ 
2.1 Multi person cooperative game theoryShapley ${ }^{[1,2,6]}$

In the ' $\mathrm{n}$ ' cooperative game $G=[N, v], G$ Shapley is an n-dimensional vector : $\Phi(v)=\left(\varphi_{1}(v), \varphi_{2}(v), \ldots, \varphi_{n}(v)\right)$ If the following axioms are satisfied :

Axiom 1: symmetry-- displace $\pi, \varphi_{\pi i}(\pi v)=\varphi_{i}(v)$

It is indicated that the distribution of ' $i$ ' in the bureau is related to the size of the contribution to the alliance, which is not related to the position in a certain arrangement.

Axiom 2:Validity--the pillar of $\mathrm{D}, \sum_{i \in D} \varphi_{i}(v)=v(D)$

Indicates that when the Shapley value is used to determine the allocation scheme, only the local people in the Bureau of the pillar D affect the allocation scheme. The dummy can be ignored in the League.

Axiom3:Additive property--Any two cooperative game $G_{1}=[N, v], G_{2}=[N, w](i \in N)$

$$
\begin{aligned}
& \varphi_{i}(v+w)=\varphi_{i}(v)+\varphi_{i}(w) \\
& (v+w)(S)=v(S)+w(S) \text { is said that in the same }
\end{aligned}
$$
game $G=[N, v]$, the income of the I through the two game is the same as that obtained by the combination of the feature function. If ' $n$ ' person cooperative game. To satisfy the above axioms, there is the value of only Shapley:

$$
\begin{aligned}
& \Phi(v)=\left(\varphi_{1}(v), \varphi_{2}(v), \ldots, \varphi_{n}(v)\right) \\
& \varphi_{i}(v)=\sum_{S \in N} \frac{(n-|S|) !(|S|-1) !)}{n !}[v(S)-v(S \backslash\{i\})]
\end{aligned}
$$

Among it, the number of $|\mathrm{S}|$ on behalf of the league players in ' $\mathrm{S}$ '. $(|S|-1)$ ! on behalf of coalition formation after S, 'I' does not contain players union arrangement number. $(n-|S|)$ ! represents the number of permutations of the League NIS. $n$ ! means that the number of permutations that $\mathrm{n}$ may form in a bureau. $\frac{(n-|S|) !(|S|-1) !)}{n !}$ represents the probability that the union $S$ appears. $v(S)-v(S \backslash\{i\})$ represents the marginal contribution of the I to the alliance S.

\section{Signal Control Method for Single Intersection based on Cooperative Game Theory}

Single intersection four phase signal is :go straight, turn left, North and south to the straight line, North and south to the left .Turn right direction of the vehicle is not controlled (As shown in Fig.1). The direction of the intersection of the main road, a totallength of 2000 meters, the north and south direction, supplemented by road, 1600 meters in length.

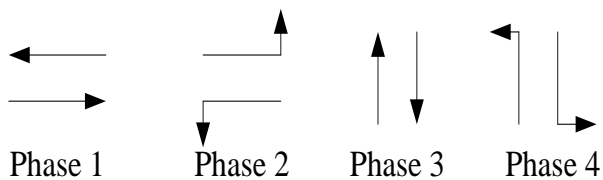

Fig.1. four phase diagram of phase signal of single intersection

\subsection{Four phase signal control game model based on single inters ection}

(1) Selection of policy set

For traffic lights, the choice of the strategy has a red light or green light（Red, Green). At the same time taking into account the existence of a red light (In addition to the short infrared ) or at the same time there is more than one direction for the green, may not be the actual intersection of single intersection signal control for four phase only 4 strategy combination of circumstances income greater than 0 that is at the same time the four phase in a phase is also light. The three phase is red. In other cases, the phase combination gains are 0.By social rules, traffic lights minimum green time and longest green time is limited.

(2) Determination of value

In the game of earned value is a predictive value, namely at the beginning of the game in a strategy to predict all the possible combinations of the proceeds. For the signal intersection control, the queue length is the most intuitive return value, so in this paper, the length of queue length is selected to win the value of the game. The calculation of the queue length is as follows 3.

$$
\begin{aligned}
& \text { queue length }=q+\text { arrive_rate } \times \text { car_length } \times t+z 1 \times t \times \text { average_speed } \\
& +z_{2} \times \text { depart_rate } \times \text { car_length } \times t
\end{aligned}
$$

In the formula, queue length indicates the value of queue length; Car_length is the average length; $\mathrm{q}$ is the current phase of the queue length; $t$ is the current phase of the execution time; verage_speed is that when the phase preserving green signal, leaving all vehicles crossing speed evaluation; arrive_rate and depart_rate is the arrival rate 
and the leaving rate. It uses the method of linear prediction to solve the fuzzy parameters. On the use of the method to determine the predicted arrival rate and the leaving rate refer to the literature ${ }^{[7]}$.

payoff $\left(\right.$ red_light $\rightarrow$ red_light or green_light $\rightarrow$ red_light), $z_{1}=0 \& z_{2}=0$

It means that when the signal is converted from a red light to a green light or a red light. Formula 4-3: $z_{1}=0, z_{2}$ $=0, \quad z_{2}=-1$

(3)The establishment of income matrix

The four phase single intersection signal control phase, get the right to pass in a moment there might be four cases, the prediction of queue length value as the game earned value in determining the income matrix will have four possible scenarios. That is, the current 4 phase lamp states are:Green,Red,Red,Red），(Red ,Green, Red, Red） ,

(Red ,Red,Green, Red) , (Red ,Red, Red, Green) ,Take the first case as an example:The current access to the current phase is the first phase. That is, the current 4 participating in the lamp state（Green,Red,Red, Red） ,In this case, the 4 participants can choose the policy portfolio, the payoff matrix of the game as shown in Table 1.

\begin{tabular}{|c|c|c|c|c|c|}
\hline & \multicolumn{4}{|c|}{ player } & \multirow{2}{*}{$\begin{array}{l}\text { payoff } \\
\text { function } \\
(\mathrm{P} 1, \mathrm{P} 2, \mathrm{P} 3, \mathrm{P} 4)\end{array}$} \\
\hline Payo & $\begin{array}{l}\text { Phase } \\
1\end{array}$ & $\begin{array}{l}\text { Phase } \\
2\end{array}$ & $\begin{array}{l}\text { Phase } \\
3\end{array}$ & $\begin{array}{l}\text { Phase } \\
4\end{array}$ & \\
\hline $\mathrm{Ff}$ & Green & Red & Red & Red & 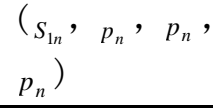 \\
\hline \multirow[t]{3}{*}{$\begin{array}{l}\text { Mat } \\
\text { rix }\end{array}$} & Red & Green & Red & Red & $\begin{array}{l}\left(p_{n}, S_{n}, p_{n},\right. \\
\left.p_{n}\right)\end{array}$ \\
\hline & Red & Red & Green & Red & $\begin{array}{l}\left(p_{n}, p_{n}, S_{n},\right. \\
\left.p_{n}\right)\end{array}$ \\
\hline & Red & Red & Red & Green & $\begin{array}{l}\left(p_{n}, p_{n}, p_{n},\right. \\
\left(S_{n}\right)\end{array}$ \\
\hline
\end{tabular}

Table 1: When the phase 1 obtains the right of way of the payoff matrix

\subsection{Solution of four phase signal control game model of single inters ection}

(1) Alliance Characteristic Function

For the 4 person cooperative game, it is possible to form the alliance:Each person in a separate act, that is, 4 of the people in the Bureau for a union; Any of the 2 members of the board of 4 members of the coalition; Any of the 3 members of the board of 4 members of the coalition; 4 people form a major league. In the income matrix shown in Table 1 , the authority of the people in the 1 as a coalition The determination method of the characteristic function $v_{a}(\{1\})$ is the alliance $S=\{1\}, N \backslash S=\{2,3,4\}$, carries on the two person zero sum game. Two person zero sum game payment matrix as shown in Table $2, \mathrm{R}$ in the table for a very large number, said in such a combination of strategies, the Union won the value that is the queue length is very large. Because in these circumstances the capacity of the intersection of each phase is very poor, so the length of each phase of the queue is very long. In the table, the payoff matrix(Red, Green ,Red ,Red), (Red, Red, Green, Red), (Red, Red ,Red, Green) is indicated in the strategy of winning the corresponding value of the alliance $S=\{1\}$.The calculation method and $p_{n}$ method are the same. The value of matrix game $v_{a}(S)=\max _{x \in X_{S}} \min _{y \in X_{N \backslash S}} \sum_{i \in S} E_{i}(x, y)$ can be got according to the formula of characteristic function $\alpha-$.The solution for the Alliance $S=\{1\} \quad$ is $: v_{a}(\{1\})=\max \left(S_{1 n}, \min \left(p_{n}{ }^{\prime}, p_{n}{ }^{\prime \prime}, p_{n}{ }^{\prime \prime \prime}\right)\right)$.

\begin{tabular}{|c|c|c|c|c|c|}
\hline \multicolumn{2}{|c|}{} & \multicolumn{4}{|c|}{ Alliance $N \backslash S=\{2,3,4\}$} \\
\cline { 2 - 6 } \multicolumn{2}{|c|}{} & $(\mathrm{R}, \mathrm{R}, \mathrm{R})$ & $(\mathrm{G}, \mathrm{R}, \mathrm{R})$ & $(\mathrm{R}, \mathrm{G}, \mathrm{R})$ & $\begin{array}{c}\text { (R, } \\
\mathrm{R}, \mathrm{G})\end{array}$ \\
\hline $\begin{array}{c}\text { Alliance } \\
S=\{1\}\end{array}$ & $\mathrm{G}$ & $S_{1 n}$ & $\mathrm{R}$ & $\mathrm{R}$ & $\mathrm{R}$ \\
\cline { 2 - 6 } & $\mathrm{R}$ & $\mathrm{R}$ & $p_{n}{ }^{\prime}$ & $p_{n}{ }^{\prime \prime}$ & $p_{n}{ }^{\prime \prime}$ \\
\hline
\end{tabular}

Table 2: alliance $S=\{1\}, N \backslash S=\{2,3,4\}$ two person zero sum game payment matrix

Calculate in a similar way, $v_{a}(\{2\}), v_{a}(\{3\}), v_{a}(\{4\})$, $v_{a}(\{1,2\}), v_{a}(\{1,3\}), v_{a}(\{1,4\}), v_{a}(\{2,3\}), v_{a}(\{2,4\})$, $v_{a}(\{3,4\}), v_{a}(\{1,2,3\}), v_{a}(\{1,2,4\}), v_{a}(\{1,3,4\})$, $v_{a}(\{2,3,4\}), v_{a}(\{1,2,3,4\}), v_{a}(\{\varnothing\})$.

At this point, the 4 person cooperative game all the possible coalition characteristic function.The following will be the solution of the valuation of the cooperative game -Shapley value.

(2)The valuation solution of multi person cooperative game: Shapley value.

Under the premise of satisfying these three axioms, the existence and uniqueness of the Shapley value for the 4 person cooperative game $G=[4, v]$ is :

$$
\Phi(v)=\left(\varphi_{1}(v), \varphi_{2}(v), \varphi_{3}(v), \varphi_{4}(v)\right)
$$


The calculation $\varphi_{i}(v), i=(1,2,3,4)$ method is as follows (2).

For multi person cooperative game model, cost allocation problem has been resolved so far, but in this paper is to solve the control problem of the actual intersection signal, so the final should be the signal control strategy combination. After finding out the Shapley value of the cooperative game, a set of strategy combination which is more close to the result of the game is selected as the final strategy combination of the game player.

\section{Simulation}

\subsection{Simulation modeling--Paramics}

Simulation model is shown in Fig.2. By social rules, set the minimum green simulation time is $20 \mathrm{~s}$. The maximum green time is $90 \mathrm{~s}$.

\subsection{Simulation result analysis}

In the Paramics simulation, the OD matrix is set to meet three different traffic levels, namely, low flow, high flow and high flow rate.

(1)Simulation under low flow rate

In general, the saturated network flow can reach $3600 \mathrm{veh} / \mathrm{h}$. On this basis, 0.3 of the coefficient of conversion of the flow rate is approximately in accordance with the standard of low flow. As shown in Table3, Zone1, Zone2, Zone3,

Zone4, represent the departure area.

\begin{tabular}{|l|l|l|l|l|l|l|}
\hline & & Zone 1 & Zone 2 & Zone 3 & Zone 4 & Total \\
\hline Zone 1 & & 540 & 540 & 100 & 1180 \\
\hline Zone 2 & 540 & & 100 & 540 & 1180 \\
\hline Zone 3 & 300 & 350 & & 150 & 800 \\
\hline Zone 4 & 350 & 300 & 150 & & 800 \\
\hline Total & 1190 & 1190 & 790 & 790 & 3960 \\
\hline
\end{tabular}

Table 3: OD matrix of low flow levels

At the time of simulation, the phase change of the intersection signal lamp is dominated by the four cooperative game algorithm. After 6 hours of simulation, the simulation evaluation data sheet can be obtained in the Paramics sub module Processor. The average speed of the total vehicle is selected as the evaluation index, and draw the simulation effect diagram in MATLAB. Compare with the optimal timing controleffect, as shown in fig.3.

(2) Simulation of medium flow rate

Medium flow rate is generally in the basis of the saturation flow rate of 0.6 of the coefficient of conversion. The OD matrix in Paramics is set in this way, as shown in Table 4.

\begin{tabular}{|c|c|c|c|c|c|}
\hline & Zone 1 & Zone 2 & Zone 3 & Zone 4 & Total \\
\hline Zone 1 & & 800 & 700 & 550 & 2050 \\
\hline Zone 2 & 800 & & 550 & 700 & 2050 \\
\hline Zone 3 & 450 & 500 & & 400 & 1350 \\
\hline Zone 4 & 500 & 450 & 400 & & 1350 \\
\hline Total & 1750 & 1750 & 1650 & 1650 & 6800 \\
\hline
\end{tabular}

Table4: OD matrix of medium flow rate

Take 6 hours in Processor simulation, get the evaluation data table, draw the simu lation effect diagram in MATLAB. And compared with the optimal timing control effect, as shown in Fig4.

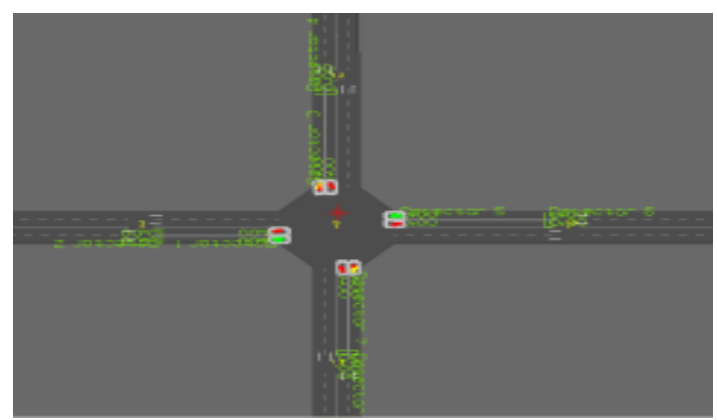

Fig.2. Model of single intersection signal control in simulation Paramics

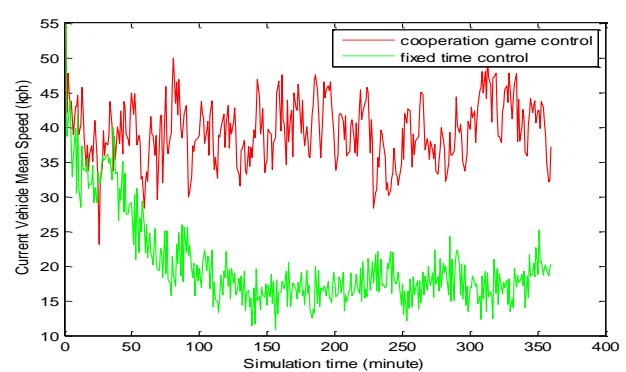

Fig.3. the effect of multi person and game control in low flow level

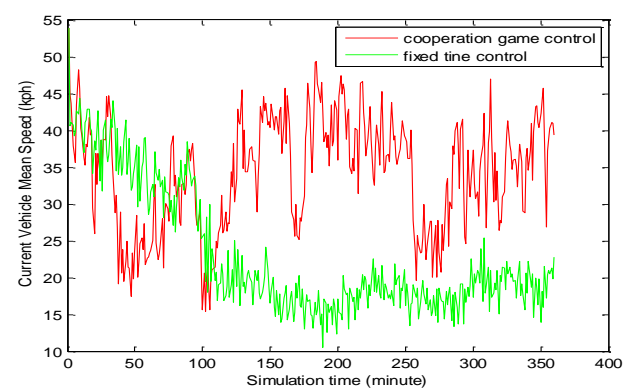

Fig.4. the effect of multi person and game control in the medium flow level 


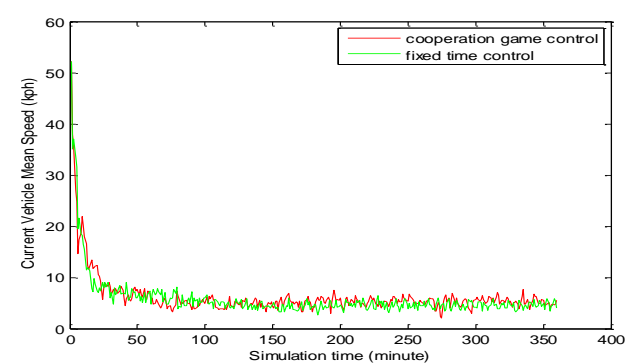

Fig.5. high traffic levels under the multi person and make game control effect chart

(3)Simulation underhigh flow rate

High flow rate is generally in the basis of the saturation flow rate of 0.8 of the coefficient of conversion. The OD matrix in Paramics is set in this way, as shown in Table 5.

\begin{tabular}{|c|c|c|c|c|c|}
\hline & Zone 1 & Zone 2 & Zone 3 & Zone 4 & Total \\
\hline Zone 1 & & 1000 & 800 & 700 & 2500 \\
\hline Zone 2 & 1000 & & 700 & 800 & 2500 \\
\hline Zone 3 & 400 & 700 & & 500 & 1600 \\
\hline Zone 4 & 700 & 400 & 500 & & 1600 \\
\hline Total & 2100 & 2100 & 2000 & 2000 & 8200 \\
\hline
\end{tabular}

Table5: OD matrix settings for high traffic levels

Take 6 hours in Processor simulation get the evaluation data table, draw the simulation effect diagram in MATLAB.And compared with the optimal timing control effect,as shown in Fig.5 .

\section{Conclusions}

In the case of low, middle and high grade of traffic class, the control method of multi person cooperative game and the optimal timing control method are carried out. Simulation effect diagram by MATLAB, through the analysis of the effect chart:

Under the low flow rate, the control method is better than the optimal timing control method, which fully embodies the idea of cooperation. In the medium flow, the control method of total vehicle average speed of road network change greatly, but on the whole is slightly better than the timing control method. Under the high flow rate, the road network traffic has exceeded the capacity of the road, no matter what kind of algorithm, can not achieve a better control effect.

\section{Acknowledgements}

This work was supported by the Quality Engineering Project of Anhuiprovince No. 2016jyxm0474、 2016jyxm0489 and Quality Engineering projects of Anhui Xinhua University No. 2015jy009、No. 2014gxkc x01

\section{References}

[1] Wang Xianyu, Xiao Yuming. Game theory and its application [M]. Beijing: Science Press, 2008

[2] Jiang Pengfei. Solution of cooperative game and its application research [D]. Shandong: Shandong University, 2007

[3] Sun Kang.N person cooperative game theory, method and its application in strategic alliance [D]. Dalian: Dalian University of Technology, 2005

[4] Zhou Xiaoling. Theory, method and application of cooperative game theory $[\mathrm{J}]$. systems engineering theory and practice, 1998 (11): 36-39

[5] Zhao Hongqiang. Solution of cooperative game and its application research [D]. Jilin: Jilin University, 2007

[6] Zhao Hongqiang. Solution of cooperative game and its application research [D]. Jilin: Jilin University, 2007

[7] Zhang Jie. Research on traffic signal lamp control algorith $m$ under the framework of game theory [D]. Beijing: Beijing University of Technology,2009 